\title{
Hill-Rom's Bed Exit System with Audible Alarm, used in Conjunction with Falls Protocol: Reduces Falls on a Medical-Surgical Inpatient Unit: A Process Improvement Project.
}

\author{
Lydia H. Albuquerque ${ }^{*}$, DNP, RN, ACNP-BC, CCRN, Bindu Jacob ${ }^{2}$, BSN, RN, Emily H. Mahon ${ }^{3}$ PhD, MBA
}

$I^{*}$ Assistant Professor, Department of Nursing, College of Science \& Health, William Paterson University, Wayne, NJ, United States.

${ }^{2}$ Registered Nurse, University Hospital, Newark, NJ, United States.

${ }^{3}$ Adjunct Professor, department of Sociology, William Paterson, Wayne, NJ, United States.

\section{Article Details \\ Article Type: Research Article \\ Received date: $22^{\text {nd }}$ January, 2020 \\ Accepted date: $15^{\text {th }}$ February, 2020 \\ Published date: $29^{\text {th }}$ February, 2020}

"Corresponding Author: Lydia H. Albuquerque, Assistant Professor, Department of Nursing, College of Science \& Health, William Paterson University, Wayne, NJ, United States. E-mail: albuquerquel@wpunj.edu

Citation: Albuquerque, L.H., Jacob, B., \& Mahon E.H. (2020). Hill-Rom's bed exit system with audible alarm, used in conjunction with falls protocol: Reduces falls on a medical -surgical inpatient unit: A Process Improvement Project. J Comp Nurs Res Care 5(1):159. doi: https://doi.org/10.33790/jenrc1100159.

Copyright: (C2020, This is an open-access article distributed under the terms of the Creative Commons Attribution License 4.0, which permits unrestricted use, distribution, and reproduction in any medium, provided the original author and source are credited.

\begin{abstract}
Background: Research is limited regarding utilization of The HillRom bed exit system with audible alarm in conjunction with the hospital protocol to reduce falls among patients admitted to inpatient units in the hospital.

Objective: To evaluate the effectiveness of an education program to improve knowledge and skills on zeroing and activation of The Hill-Rom bed exit system with audible alarm in conjunction with hospital protocol to reduce falls rate on a medical-surgical unit of an acute care hospital.
\end{abstract}

Method: A quantitative, descriptive correlational design $(\mathrm{n}=32)$ was used to examine the effect of an education program and skills on the pre and post-test scores and post-intervention skills on zeroing and activation of The Hill-Rom bed exit system with audible alarm to falls rate on a medical-surgical unit.

Results: A sample t- test was used to examine the effectiveness of education on pre-test and post-test scores. The post-test mean was 2.65 greater, $\mathrm{t}=8.048, \mathrm{p}=.000$. The results were statistically significant with gain of knowledge and skills post intervention. This gain in knowledge and skills was directly related to a $55 \%$ decrease in falls rate from 5.8 to 3.2 per 1,000 patient days. A one-way ANOVA examined the difference in means between the knowledge gained of the registered nurses and certified nursing assistants. There was no significant difference within and between groups.

Conclusion: Staff education and continued use of The Hill-Rom bed exit system with audible alarm along with the hospital protocol were associated with decrease in falls rates on a medical surgical unit of an academic medical center.

Key words: Falls, Bed Exit Alarm, Setting, Zeroing, Hill-Rom

\section{Introduction}

Falls are a growing public health concern and a source of injuries for older adults. Each year approximately 1 in 5 older adults in Untied States experience a fall [1]. Every year, 3 million emergency department visits are related to falls [1]. Fall death rates among adults age 65 and older has increased more than $3.0 \%$ per year during 2007 to 2016 [2]. In 2015, \$50 billion was spent on fatal and non-fatal falls injuries and $\$ 754$ million is spent on fatal falls [3, 4]. Medicare and
Medicaid shouldered $75 \%$ of these costs [1]. Reduced activity, chronic condition, increased use of prescription medication and agerelated gait and balance problems are factors attributed to falls [3].

Falls prevention programs incorporate multiple components that requires involvement of a multi-disciplinary team $[4,5]$. One step that healthcare facilities are implementing to reduce the number of accidental patient falls is using standardized assessment tools to gauge a patient's risk for falling [6]. These tools alone have not proven to decrease the number of falls causing fatal and nonfatal injuries [4]. Hill-Rom developed smart beds designed that transmit data to a call system. When a movement out of the bed is detected, the call system notifies the nurse through an alarm sound and call light. This alarm can be connected to a smart bed which also has an automated documentation program. The reliability of using bedexit alarm alone in preventing falls and injury of falls have not been established. Some studies reported no statistical significance in whether bed alarms alone prevent falls [8]. A systematic review on inpatient fall prevention programs concluded that inpatient falls can be reduced by including risk assessment, staff education, bedside signs, and utilizing a multi-disciplinary approach with leadership support [9]. A study conducted evaluated the effectiveness of a patient education video and visual signaling icons added to bed exit alarms. The study findings were associated with significant reduction of falls and fall related injuries [10].

This process improvement project was aimed at implementing the utilization of the Hill-Rom bed exit system with audible alarm, in conjunction with the hospital protocol. The goal was to reduce patient falls related to unsupervised bed egress on a medical-surgical unit of a large academic medical center

\section{Background}

In a large academic medical center, the falls rate and injuries were trending upwards. The falls prevention committee instituted falls protocol which included Morse falls risk assessment tool, falling stars, high risk patients' yellow bands and hands-off shift report. The falls rate on the medical-surgical unit continued to be at an all-time high in spite of efforts made by the falls prevention committee. In 2015, the Hill-Rom Versa Care beds were launched at the academic medical center, nurses and certified nursing assistants were educated to zero 
beds and activate the Hill- rom bed exit system with audible alarm. Hill-Rom's bed exit system with audible alarm is zeroed and activated by the registered nurse or certified nursing assistant. Zeroing of bed is an important feature to be initiated prior to patient being placed on the bed. Bed alarms are activated for patients with a Morse score $\geq 46$.

The Morse Fall Scale (MFS) is often used by registered nurses in conjunction with the patient's clinical assessment and medication list [11]. In the MFS, the patient's history of falling, secondary diagnoses, use of ambulatory aid, IV access, gait, and mental status are assessed and scored. The amount of points acquired in each section is dependent on the patient's response. Patients who score 0-24 on the MFS are at no risk for falls. Patients who score between 25-45 on the assessment are at a low-moderate risk for falling and it is recommended that standard fall prevention interventions are implemented. Patients who receive a score of 46 or higher on the MFS are at a high risk for falling. In the hospital setting high risk falls prevention interventions are implemented by assessing a patient's risk for falling with a risk assessment tool upon hospital admission. Healthcare professionals can be ahead of patient falls because they will know what preventive interventions to implement in order to decrease the incidence of patient falls.

The high falls rate on this unit prompted the investigators to explore further interventions to improve and reduce falls rate. The investigators of the study made a spot check to examine if all HillRom bed exit system with audible alarm were zeroed, activated and used in conjunction with the hospital falls protocol on patients with high Morse scores as indicated in the hospital falls prevention policy.

The spot check revealed that 14 patients had a Morse score $\geq 46$, only $4(25.57 \%)$ bed alarms were activated. This finding prompted the investigators to implement this process improvement project, where by the registered nurses and certified nursing assistants were educated on zeroing of bed and activating Hill-Rom bed system exit with audible alarm along with the falls protocol instituted by the hospital fall prevention committee and policy.

\section{Purpose of the Study}

The purpose of this project was to examine the effectiveness of an education intervention \& skills demonstration based on recommendation of Hill-Rom bed exit system with audible alarm on falls rate on a medical surgical unit of an academic medical center. The independent variables in this study were zeroing and activating The Hill-Rom bed exit system with audible alarm and the dependent variable was falls rate.

A PICOT question was developed to study the effectiveness of the education and skils intervention on zeroing and activation of bed alarm along with the hospital falls prevention protocol to falls rate. The population $(\mathrm{P})$ consisted of registered nurses and nursing assistants. The Intervention (I) consisted of educating and skills demonstration on zeroing the bed and activating The Hill-Rom bed exit system with audible alarm. The outcome $(\mathrm{O})$ examined the falls rate on the unit post intervention. Time $(\mathrm{T})$ was measured during a one-yeartime frame.

The research question that guided the study was, what is the association between education intervention and skills demonstrationon falls rate on a medical-surgical unit of an academic medical center?

\section{Methods}

\section{Study design}

A quantitative, descriptive correlational design was used. The participants $(n=32)$ was used. This methodology was used to determine the effectiveness of an educational intervention and skills demonstration on zeroing of bed and activation of The Hill-Rom bed exit system with audible alarm along with hospital fall prevention protocol on falls rate on a medical-surgical unit of an academic medical center.

\section{Ethical Consideration}

Hospital research committee approval was obtained from the participating study site prior to implementing the study.

\section{Setting and Sample}

A purposive sampling technique was utilized. Male and female registered nurses and certified nursing assistants working on the medical-surgical unit at an academic medical center were recruited. Only registered nurses and certified nursing assistants who were full time employees were included in the study. All participants able to read and write in English and willing to participate in the study were included.

\section{Data Collection}

Nineteen registered nurses and thirteen certified nursing assistants working on the medical surgical unit of a large academic medical center participated in the study that started in November 2016. The manager, registered nurses and certified nursing assistants were informed about the study. The researchers personally explained the study purpose to the participants and written consent was obtained. Participants ability to zero beds and activate Hill-Rom bed exit system with audible alarm was validated prior to the education intervention with a skills check list by the investigators.

A pre-test to assess the knowledge of participants on items related to zeroing of bed and activating The Hill-Rom bed system with audible alarm was administered with an eight-item questionnaire. The eightitem questionnaire was independently completed by each participant. An education program designed and recommended by Hill-Rom, was offered to all participants individually over 60 minutes during which the zeroing and activation of bed alarms was demonstrated to each participant. Following the completion of the education intervention and skills demonstration a post test was administered to all the participants and the skills were revalidated in zeroing of bed and activation of The Hill-Rom bed exit system with audible alarm. The falls rate was monitored after all participants received education and skills demonstration on zeroing of bed and activation of Hill-Rom bed system with audible alarm, which was completed in Dec 2016.

\section{Instruments}

An eight-item questionnaire was developed by the investigators to assess the knowledge of the registered nurses and certified nursing assistants to zero beds and activate Hill-Rom bed exit system with audible alarm. The items on the questionnaire included Yes/ No responses. The questions included responses of how to zero a bed, when to zero a bed, when to activate the bed alarm, who to call if the bed is not functioning for replacement, hospital policy on Morse scores to set the bed alarm. One question on the questionnaire assessed the registered nurses and certified nursing assistants knowledge on replacing a non functional bed on the unit. A skills validation check list was utilized to check the skills of the participants to activate the bed alarm. The participant skills to zero bed and activate bed alarm was validated by the investigators of the study.

\section{Statistical Analysis}

The international Business Machine (IBM) Statistical Package for Social Science (SPSS) statistical software version 23.0 (IBM Corp, Armonk, NY) was used to analyze the clinical and demographic characteristics. Descriptive statistics such as frequencies, percentage, mean and median were used to describe the demographic and clinical characteristics of the participants which included age, gender, qualification, years of service, level of education and category of licensure preparation. A t- test was used to examine the effectiveness of the education on the pre-test and post-test scores. A one-way ANOVA examined the difference in means between the scores of the registered nurses and certified nursing assistants.

\section{Results}

\section{Demographic Characteristics of the Study participants}

A total of 32 participants recruited to this study completed all study related pre-test, post-test assessments, 60 minutes education intervention and skills in zeroing bed and activating the Hill-Rom bed 


\begin{tabular}{|c|c|c|}
\hline Variable & $\mathrm{N}$ & $\%$ \\
\hline \multicolumn{3}{|l|}{ Category of participants } \\
\hline Registered nurses & 19 & 59.38 \\
\hline Certified nursing Assistants & 13 & 40.62 \\
\hline \multicolumn{3}{|l|}{ Gender } \\
\hline Female & 31 & 96.9 \\
\hline Male & 1 & 3.13 \\
\hline \multicolumn{3}{|l|}{ Age in Years } \\
\hline $20-30$ & 9 & 28.12 \\
\hline $31-40$ & 12 & 37.50 \\
\hline $41-50$ & 10 & 31.25 \\
\hline $51-60$ & 1 & 3.13 \\
\hline \multicolumn{3}{|l|}{ Years of experience } \\
\hline $0-5$ years & 12 & 37.50 \\
\hline $6-10$ years & 9 & 28.12 \\
\hline $11-15$ years & 10 & 31.25 \\
\hline $16-20$ years & 1 & 3.13 \\
\hline \multicolumn{3}{|c|}{ Education Qualification of registered nurses } \\
\hline Master's in nursing & 2 & 6.25 \\
\hline Associate degree & 6 & 18.75 \\
\hline Bachelor's in nursing & 11 & 34.37 \\
\hline
\end{tabular}

exit system with audible alarm. Majority of the participants (59.38\%) were registered nurses while $(40.62 \%)$ were certified nursing assistants. The participants were predominately female (96.9\%). Majority of the registered nurses (34.37\%) had a bachelor's degree in nursing. The participant's years of experience varied from 5 years to 15 years $(96.87 \%$ ) (see Table 1$)$

\section{Morse score and setting of bed alarm}

The investigators made a spot check to determine if the bed alarms were activated on patients with a Morse score $\geq 46$. Prior to the education intervention 14 patients had a Morse score $\geq 46$, only 4 $(25.57 \%)$ bed alarms were activated. Post education intervention and skills demonstration found nine patients had a Morse score $\geq 46$ and $100 \%$ of the bed alarm were activated (see Table 2).

\begin{tabular}{|l|l|l|l|}
\hline Variable & Number of patients with Morse Score $\geq 46$ & Alarm on & Percentage \\
\hline Pre intervention & 14 & 4 & $28.5 \%$ \\
\hline Post intervention & 9 & 9 & $100 \%$ \\
\hline
\end{tabular}

Table 2. Comparison of activation of bed alarm Pre-intervention and Post-intervention

\section{Pretest/ Post test scores on zeroing and activation of bed alarms}

The mean educational score of the pre-test was 4.94 , and the mean score on the post-test was $7.56(0-8)$. This resulted in a $42 \%$ increase in test scores post educational intervention (see Table 3). The eightitem questionnaire included responses of how to zero a bed, when to zero a bed, when to activate the bed alarm, who to call if the bed is not functioning for replacement, hospital policy on Morse scores to activate the bed alarm.

A sample t-test was used to examine the effectiveness of education on pre-test and post -test scores. The post-test mean was 2.65 greater, $\mathrm{t}=8.048, \mathrm{p}=.000$. The results were highly significant with gain of knowledge and skills post education intervention. There was no difference in the pre-test/ post-test scores in relation to education qualification of the participants (see Table 3, 4, 5).

\begin{tabular}{|l|l|l|l|l|}
\hline & $\mathrm{N}$ & Mean & Std deviation & Std Error mean \\
\hline Pre-test score & 32 & 4.94 & .716 & .127 \\
\hline Post-test score & 32 & 7.56 & 1.722 & .304 \\
\hline
\end{tabular}

Table 3. Comparison of test scores Pre-intervention and Post-intervention

\begin{tabular}{|l|l|l|l|l|l|}
\hline & $\mathrm{N}$ & Mean & Std. Deviation & Minimum & Maximum \\
\hline${ }^{*}$ CNA & 13 & 2.7692 & 2.08782 & 1.00 & 7.00 \\
\hline Associate Degree & 7 & 2.7143 & 1.25357 & 1.00 & 5.00 \\
\hline BSN/MSN & 12 & 2.2727 & 2.00454 & -2.00 & 5.00 \\
\hline Total & 32 & 2.5806 & 1.85785 & -2.00 & 7.00 \\
\hline
\end{tabular}




\begin{tabular}{|l|l|l|l|l|l|}
\hline Variables & Mean & Std. Deviation & $\mathrm{t}$ & $\mathrm{df}$ & Sig (2-tailed) \\
\hline Pre-test-Post test scores & 2.625 & 1.845 & 8.049 & 31 & $.000^{*}$ \\
\hline
\end{tabular}

Table 5. Relationship between Pre and Post test scores

Skills validation of Zeroing of beds and activation of bed alarms

All participants were tested for zeroing and activation of the bed alarm. Pre-intervention, 17 (53.1\%) of participants were able to zero the beds and activate the bed alarm. Post-intervention all participants
$32(100 \%)$ were able to zero the bed and activate the bed alarm, indicating that participants $15(46.9 \%)$ were further educated through a skills demonstration of zeroing and activation of bed alarms (See Table 6 )

\begin{tabular}{|c|c|c|}
\hline & Frequency & Percent \\
\hline \multicolumn{3}{|c|}{ Pre- Intervention } \\
\hline Yes & 17 & 53.1 \\
\hline No & 15 & 46.9 \\
\hline \multicolumn{3}{|c|}{ Post- Intervention } \\
\hline Yes & 32 & 100 \\
\hline No & & \\
\hline
\end{tabular}

Falls rate

The fall rate in the unit was monitored and compared from days.

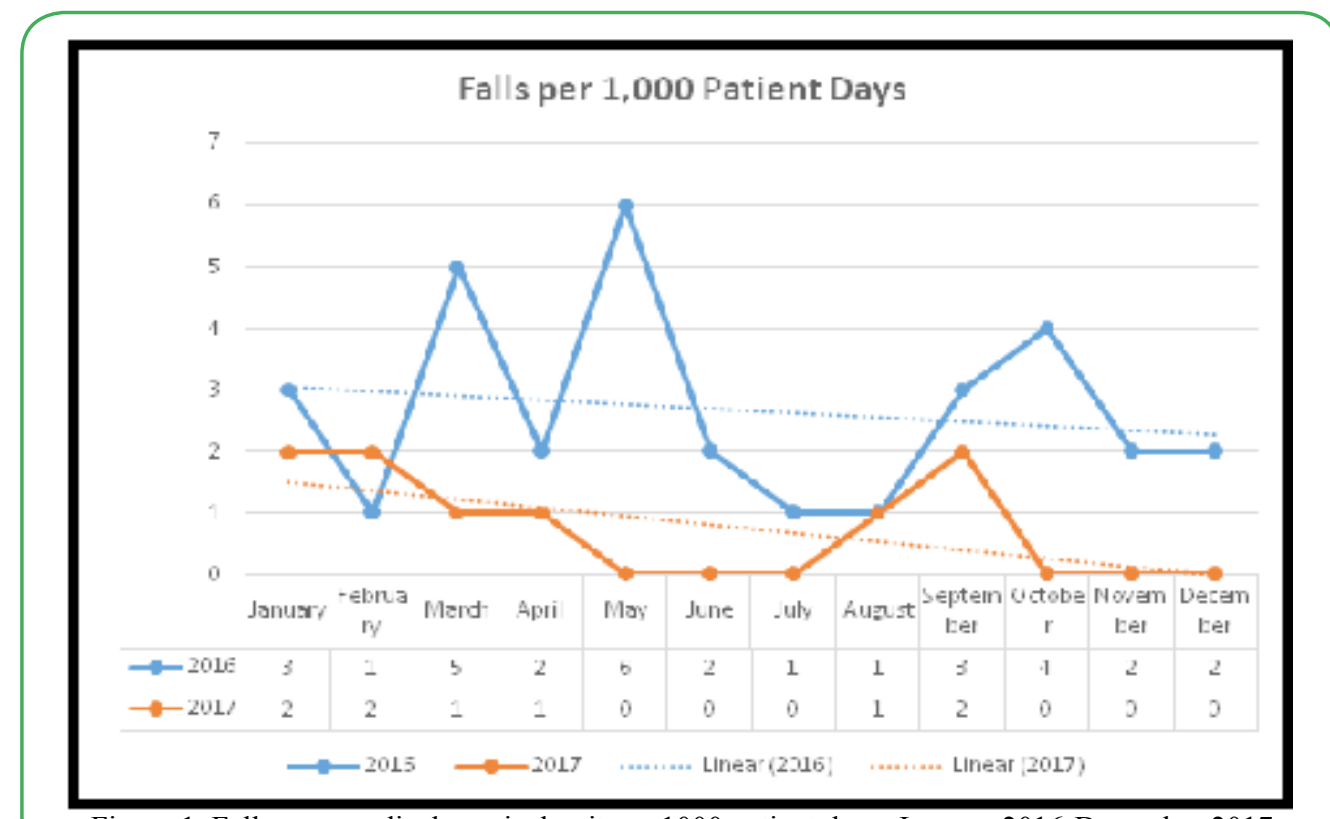

Figure 1. Falls on a medical-surgical unit per 1000 patient days, January 2016-December 2017

\section{Discussion}

This study investigated the association between an education intervention and skills demonstration on falls rate on a medicalsurgical unit of an academic medical center. In this study majority of the participants $(59.38 \%)$ were registered nurses while $(40.62 \%)$ were certified nursing assistants. The participants were predominately female (96.9\%). Majority of the registered nurses (34.37\%) had a bachelor's degree in nursing. The participant's years of experience varied from 5 years to 15 years $(96.87 \%)$.

\section{Falls rate}

The fall rate in the unit was monitored and compared from January 2016 to December 2017. Falls decreased 55\% from 5.8 to 3.2 per 1000 patient days (PDs). The decrease in falls rate can be attributed to the gain in knowledge and skills in zeroing of beds and activation of the bed alarms on patient with Morse scores $\geq 46$ in conjunction with hospital falls protocol. These findings are supported by previous research study that utilized videos, icons and alarms to educate patients at high risk for falls. Visual signaling demonstrated a reduction in fall rates by $20 \%$ from 4.78 to 3.80 per 1000 PDs, falls with injury decreased $40 \%$ from 1.01 to 0.61 per 1000 PDs and falls with serious injury $85 \%$ from 0.159 to 0.023 per 1000 PDs [10].

\section{Education intervention}

The eight item questionnaire tested the participant's knowledge with regards to zeroing of bed and activation of bed alarm. The mean score of the pretest was 4.94, and the mean score on the post test was 7.56. This is reflective of a $42 \%$ increase in test scores post educational intervention. These findings are supported by a study that utilized Knowles principle of andragogy to engage staff in the development and implementation of the Certified Fall Prevention Advocate (CFPA) program on the medical surgical unit. The falls rate decreased from 1.115 to 0.178 per 1000 PDs over a 1 -year period [12].

\section{Zeroing and activation of bed alarms}

In order for the bed alarms to be set, it is important that the bed is zeroed and activated. The findings of the study revealed that the participants had knowledge gaps in zeroing and activation of bed 
and required to be coached to zero and activate bed alarms. This finding is supported by a study in which participants attended educational calls, coaching through a virtual break through series. The common interventions included staff education, post fall huddle and intentional rounding. Outcomes were tracked for six months. Falls injury decreased from 7.4 to 5.6 per 100 census days [13]. The study findings support the previous researches on education intervention on reducing falls in inpatient unit in the hospital.

\section{Conclusion}

Hill-Rom's bed exit system with audible alarm, used in conjunction with a falls protocol, was found to decrease the fall rates on a single medical surgical unit. Falls decreased 55\% from 5.8 to 3.2 per 1,000 patient days from 2016-2017. The zeroing of beds and activation of bed alarms among patients with Morse score $\geq 46$ increased from $28.5 \%$ to $100 \%$. Nurses and certified nursing assistants require consistent education, and spot checks to ensure that Hill-Rom bed system with audible alarm are utilized to benefit patients in reducing falls that could be fatal and nonfatal. Clinical care is essential and an important component to prevent falls. Scaling up initiatives at unit level can help in improving the health of patients at high risk for falling. Preventive strategies can lead to a substantial reduction in health care spending. New employees require to be oriented to The Hill- Rom bed exit system with audible alarm.

The results of this study were shared with the hospital process improvement council and hospital leadership team. The department of nursing education and professional development was engaged in changing policies required for enhancing practice to include education along with skills validation on utilization of The HillRom bed exit system with audible alarm at the yearly mandatory competency for registered nurses and certified nursing assistants.

\section{Limitation}

The limitation of the study was the small sample size $(n=32)$ and the study participants were from a single unit, hence the findings cannot be generalized to all patients in the hospital. There were several interventions that were implemented and hence it is difficult to state if the education intervention on setting and zeroing of The Hill-Rom bed exit system with audible alarm was the only contributing factor to reduce the falls rate in this study.

\section{Future Research}

Multi-center randomized trials are needed to confirm the effectiveness of The Hill-Rom's bed exit system with audible alarm, used in conjunction with falls protocol. A larger sample including different floors in the hospital should be considered.

Conflicts of Interest: The authors declare no conflicts of interest.

\section{References}

1. Centers for disease control and prevention (CDC). National center for health statistics. (2020). Fall facts. Retrieved from https//www.cdc.gov//fall/adultfalls.html.

2. Burns, E., \& Kakara, R. (2018). Deaths from falls among persons aged $\geq 65$ Years- United States, 2007-2016. Morbidity and Mortality Weekly Report 67(18), 509-514. doi: http:// dx.doi.org/10.15585.

3. Florence, C. S., Bergen, G., Atherly, A., Burns, E., Stevens, J. and Drake, C. (2018). Medical costs of fatal and nonfatal falls in older adults. J Am Geriatr Soc, 66(4), 693-698. doi: 10.1111/jgs.

4. Haddad, Y. K., Bergen, G., Florence, C.S. (2019). Estimating the economic burden related to older adult falls by state. $\mathrm{J}$ Public Health Managed Pract.25(2):E17-E24. doi:10.1097/ PHH.0000000000000816.

5. Dibardino, D., Cohen, E. R., Didwania, A. (2012). A metaanalysis: Multidisciplinary fall prevention strategies in the acute care inpatient population. J Hosp Med, 7(6):497-503. doi: 10.1002/jhm.1917.
6. Dykes, P. C., Carroll, D. L., Hurley, A. C., Benoita, A. Middleton, B. (2009). Why do patients in acute care hospitals fall? Can falls be prevented? J Nurs Adm, 39(6):299-304. doi: 10.1097/ NNA.0b013e3181a7788a.

7. Cruz, S., Carvalho, A. L., Barbosa, P. \& Lamas, B. (2015). Morse fall scale user's manual: Quality in supervision and in nursing practice. Procedia - Social and Behavioral Science 171, 334-339. doi: 10.1016/j.sbspro.2015.01.130.

8. Shorr, R. I., Chandler, A. M., Mion, L. C., Waters, T. M., Liu, M., Daniels, M. J., Kessler, L. A. \& Miller, S. T. (2012). Effects of an intervention to increase bed alarm use to prevent falls in hospitalized patients: A cluster randomized trial. Annals of Internal Medicine, 157(10): 692-699. doi:10.7326/0003-4819157-10-201211200-00005.

9. Miake-Lye, I.M., Hempel, S., Ganz, D.A., Shekelle, P.G. (2013). Inpatient fall prevention programs as a patient safety strategy: A systematic review. Ann Intern Med. 158:390-396. doi: https:// doi.org/10.7326/0003-4819-158-5-201303051-00005.

10. Cuttler, S. J., Barr-Walker, J., \&Cuttler, L. (2017). Reducing medical-surgical inpatient falls and injuries with videos, icons and alarms. BMJ open quality, 6(2), e000119. doi:10.1136/ bmjoq-2017-000119.

11. Pasa, T. S., De Souza Magnago, T. S., Urbanetto, J. D., Baratto, M. A., Morais, B. X., \& Carollo, J. B. (2017). Risk assessment and incidence of falls in adult hospitalized patients. Rev. Latino-Am. Enfermagem, 25, e2862, doi: 10.1590/15188345.1551.2862.

12. Reich, J., Farrell, K., Maloney, C., Drayton, D., \& Johnson, T. (2017). Decrease falls with injury. Journal of Nursing care Quality, 32 (1), 40-46. https://doi-10.1097/NCQ000000000000026.

13. Zubkoff, L., Neily, J., Delanko, V., Young-Xu, Y., Boar, S., Bulat, T., \& Mills, P.D. (2019). How to prevent falls and fallsrelated injuries: A virtual breakthrough series collaborative in long term care. Physical and Occupational therapy in geriatrics, 374 (4), 234-246. https://doi-org/10.18080/02703181. 